\title{
Unconventional Machining Process: Magnetorheological Fluid-Based Surface Finishing
}

\author{
Saurabh Pathak ${ }^{1,2 *}$ and Rajni Verma ${ }^{1,3}$ \\ ${ }^{1}$ School of Engineering, RMIT University, Australia
}

${ }^{2}$ Department of Mechanical Engineering, University of Melbourne, Parkville, Australia

${ }^{3}$ School of Physics, The University of Melbourne, Australia

*Corresponding author: Department of Mechanical Engineering, University of Melbourne, Parkville, VIC, 3010 Australia

\begin{abstract}
The development of new material with significantly improved properties, miniaturization of devices and complex design necessities in numerous domains of engineering, demands high productive, efficient and precise machining processes. Unconventional machining process (UMP) or non-traditional machining process provides solutions to these challenges and has extended their utility for the machining of complex shapes precision machining (micro-nano finishing), newly developed materials (alloys, composites, polymers, metals, rubber etc.). UMP have shown potential in the domain of micro-nano machining and provides better proposition with numerous intrinsic recompences which makes UMP a suitable substitution of the conventionally used machining process. The opportunities challenge, and recent developments of MRF based UMP method have been studied including several pragmatic applications in diverse manufacturing and industrial domains. This UMP techniques can further be modified to utilized it at its full potential in various engineering applications.
\end{abstract}

\section{Introduction}

The current progression in engineering technologies demands high precision quality manufacturing system whit high productivity to meet the current requirements of the industries. This creates a distinctive opportunity for the developments of novel and efficient machining processes which meets the specific criteria for the fabrication of desired products. The development of new materials with significantly improved properties such as hardness, strength, toughness, flexibility etc. are difficult to process with conventional machining processes $[1,2]$. UMP provides the prospect to produce components and shapes of relatively new materials having complex design requirement with precise dimensional features and parameters. The rapid growth of hybrid materials and miniaturization of devices high precision defect free machining is recommended to meet the desired efficiency. Composites and alloys with greater physical properties like toughness, tensile strength compressive strength, resilience etc. has gained lots of popularity as it provides an efficient solution for the needs to the present-day demand in numerous fields like applied thermo power and storage, biotechnology and food processing, power generation, transportation, material synthesized, etc. Various conventional manufacturing techniques have been employed such as turning, shaping, lapping, honing, blanking etc. are used in machining which utilizes a high strength cutting tool with specified tool geometry. These processes are limited because of their lower rate of material removal (MRR), lower precision, tool wear etc. To overcome these challenges unconventional or advanced machining processes arose which does not use any material and energy in its direct form is utilized for machining. Figure 1 shows the general classification of the classification of the machining process listing various machining techniques [3].

\section{Unconventional Machining Process}

UMPs are also termed as advanced machining processes or non-conventional machining process but looking at their spectrum of various uses in small and medium scale industries in last decade, non-conventional or non-traditional termed is faded off and UMPs 
are now termed as advanced machining process (AMP). UMP technique can be defined as the machining process which does not require any sharp cutting-edge tool for material removal from work piece. It utilizes thermal, mechanical, electrical, chemical, magnetic energy or combination of these energy for material removal. In conventional or traditional machine process like milling, turning etc. require workspace clamping which are not easy for the irregular and complex shapes. Also, the surface-finish obtained with these processes are not satisfactory for various sophisticated mechanical components with high precision requirements. Traditional methods are not appropriate to machine extremely firm, fragile and inelastic material whereas UMP suits perfect for these requirement as they use direct energy, so problem encountered in conventional methods regarding the tool failure does not occur with these methods [3].

Component with intricate and complex three-dimensional shapes can also be machined with these non-traditional methods. The main advantages of these processes are higher material removal rate i.e. high productivity, no residual stress, workpiece clamping is not required, better quality, less Material wastage, and complex geometries \& delicate part can easily be machined. A few UMP processes have been invented based on the specific requirement of the devised product. UMP techniques can be mainly classified in three different classes founded on the energy used for material removal; mechanical, thermal and chemical \& electrochemical. The material removal mechanism is entirely different in UMP, as traditional methods involves force application on the workpiece with a cutting tool and direct physical contacts between work piece and tool take place whereas in UMP various forms of energy are used which replaces the cutting tool [4].

\section{Classification of Unconventional Machining Process}

Machining are generally pronounced as the material removal from the workpiece through cutting, using single/multi-point cutting tools, grinding and UMP utilized by various energy sources. The main categories of the machining methods are shown in Figure 1. To control the major problems encountered in the processing of the novel and complex material and shapes, tools are usually made of advanced materials which are driven by novel and cuttingedge methods. Furthermore, the conventional tools of machining methods and processes be contingent by different methods drilling, sawing, broaching, etc. Although, conventional methods similar to forming be contingent on energy from electrical motor, hydraulics and gravitational forces. Moreover, the UMPs are not pretentious by mechanical behavior of novel and complex material which can produce multifaceted profiles by governing diverse processing characteristics of the methods. UMP methods can broadly categories in two sub sections, micromachining and micro-finishing. Various non-Traditional machining processes with their appropriate classification has been depicted in Figure 2 [4].

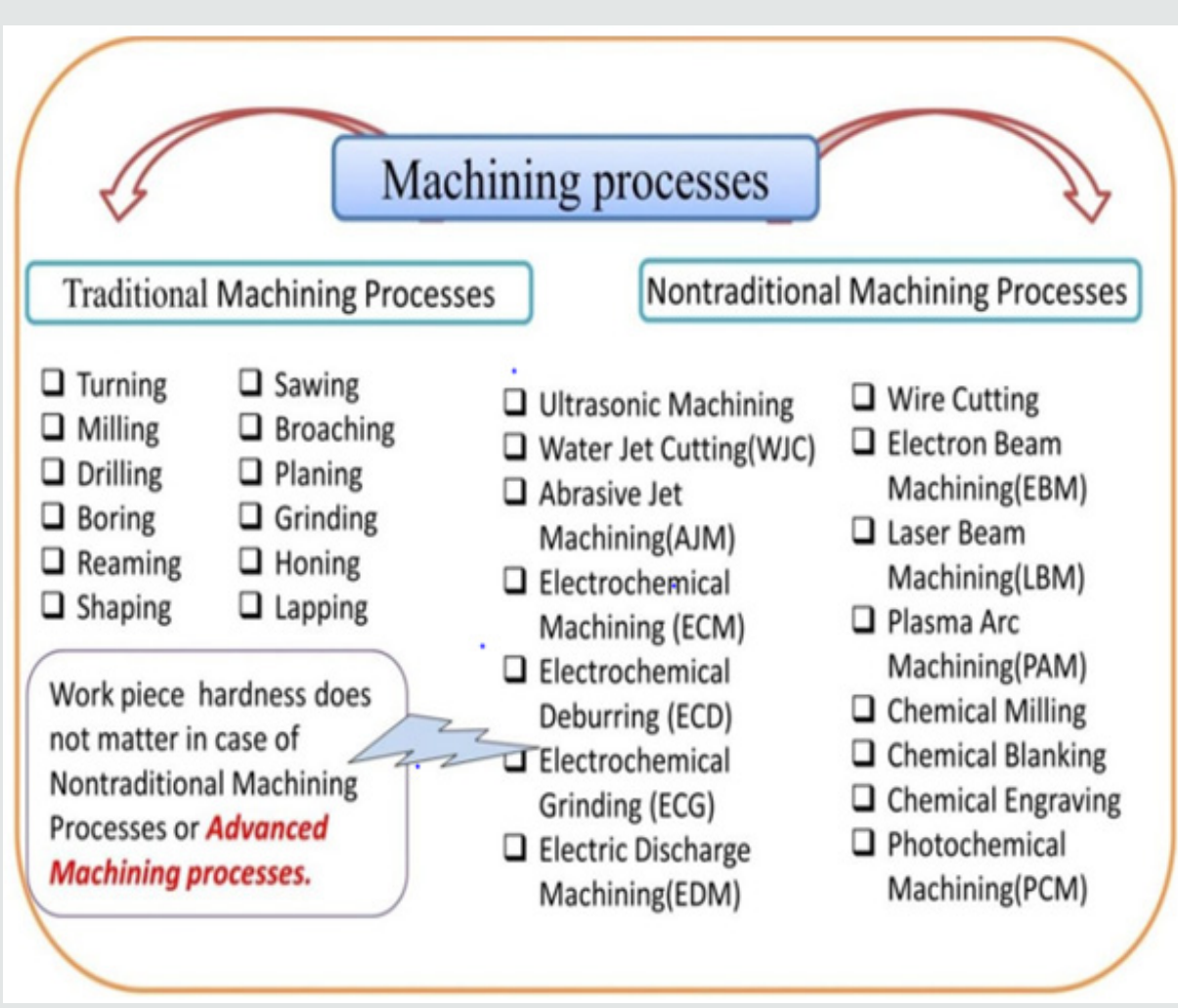

Figure 1: Classification of Machining processes. 


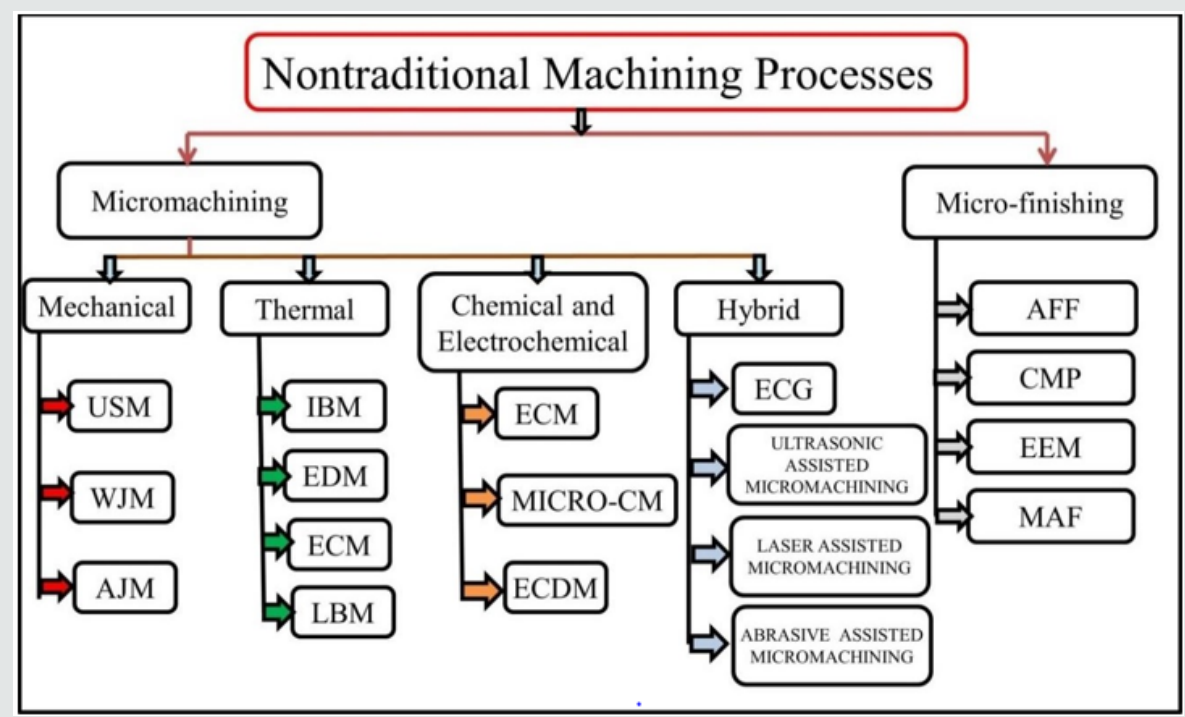

Figure 2: Classification of unconventional machining process.

\section{Magnetorheological Fluid (MRF) Based Surface Finishing}

One of the most popular UMP finishing methods is abrasive flow (AF) machining which is founded on the abrasive-slurry based working media conceded over the workpiece through channels or nozzles or fitting assemblies, were presented to retort to the cumulative industry demands. The particles of the abrasive constituent suspended in the slurry ignoble stream of (AF) machining performance as multi- movement/cutting boundaries upsetting the roughness peak. Further, to improve the (AF) machining performance and make them more controllable, the MRF based machining was introduced. MRF based machining is a novel micro/nano finishing processes which involves rotation of the workpiece and daunting an external magnetic field using MR fluid as working fluid providing assistance for the manipulations and improvement of surface finish due to enlarged applied forces on the workpiece [5-13]. In the same way, twirling the inlet flow by fixed twirler blades, is castoff which also intensifies the workpiece surface. forces and then increase the material removal rate which is having lower outlay and energy ingesting associated with the condition of rotation of the workpiece $[6,9,11]$.

The working fluid termed MRF belongs to the category of the of advanced material or smart fluids which can be utilized to regulate the machining by daunting an external magnetic field. For finishing operation, distinctively an MRF containing abrasive particles of the micron sizes and magnetic particles are synthesized with paraffin as the liquid medium and grease oil to separate magnetic particles from each other to enhance the stability. So, MRF can be tuned by an externally applied magnetic field which delivers large normal and tangential forces on the workpiece surface by magnetic particles. By applying an external magnetic field, MRF presents non-Newtonian characteristics displaying viscoelastic behavior [5-7,10,12].
MRF based surface finishing operations for high precision machining employs abrasive mixed slurry mixture of dine abrasive particles and MRF to achieve micron level finishing. The use of the MRF in the slurry is done to guide the nozzle more precisely to follow a specified path which in turn will help in improving the control over the process of machining. The mechanism of material remove in this process is propagated by the impact of the abrasive particles on the uneven surface of the workpiece which is directed by the gradient magnetic field $[5,6,9,12,13]$.

\section{Conclusion}

The current development in the field of micro/nano manufacture method leads to invention of unique and complex topographies and entities in micro/nano-meter for advanced applications. Although these features cannot be made through the conventional machining process which leads to the development of the novel UMP machining methods. UMP uses the machining of provides major advantages for processing and fabricating 3D complex shapes and nano/micro level finishing which is not economical or feasible by conventional method. MRF based finishing is UMP process which is gaining lots of popularity as it is more controllable and provides better surface finish over the conventional process. These techniques have tremendous potential to substitute the current surface finishing methods. The present mini review of the UMPs provides an overview of the various UMP methods and prospects of the MRF based finishing method.

\section{References}

1. Alam Z, Khan DA, Jha S (2019) MR fluid-based novel finishing process for nonplanar copper mirrors. The International Journal of Advanced Manufacturing Technology 101(1): 995- 1006.

2. Youssef AH (2015) Nontraditional Machining Processes-an Overview. in Machining of Stainless Steels and Super Alloys pp. 141-177. 
3. Jahan M (2019) Electro-Discharge Machining (EDM). Modern Manufacturing Processes pp. 377-409.

4. Zhong ZW (2008) Recent Advances in Polishing of Advanced Materials. Materials and Manufacturing Processes 23(5): 449-456.

5. Singh A (2020) Tuning the magneto crystalline anisotropy and spin dynamics in CoxZn1-xFe204 $(0 \leq x \leq 1)$ nano ferrites. Journal of Magnetism and Magnetic Materials 493: 165737.

6. Jahan N (2017) Enchantment in viscoelastic properties of flake-shaped iron based magnetorheological fluid using ferrofluid. Colloids and Surfaces A: Physicochemical and Engineering Aspects 529: 88-94.

7. Jain K (2019) Dynamic magneto-optical inversion in magnetic fluid using NanoMOKE. Journal of Magnetism and Magnetic Materials 475: 782-786.

8. Jain K, Pathak S, Pant RP (2016) Enhanced magnetic properties in ordered oriented ferrofibres. RSC Advances 6(75): 70943-70946.
9. Mishra A (2019) Measurement of Static and Dynamic MagnetoViscoelasticity in Facile Varying pH Synthesized CoFe204-Based Magnetic Fluid. IEEE Transactions on Magnetics 55(12): 1-7.

10. Noorjahan (2017) Dipolar Interaction and Magneto-Viscoelasticity in Nanomagnetic Fluid. Journal of Nanoscience and Nanotechnology 18(4) 2746-2751.

11. Noorjahan (2018) Improved magneto-viscoelasticity of cross-linked PVA hydrogels using magnetic nanoparticles. Colloids and Surfaces A: Physicochemical and Engineering Aspects 539: 273- 279.

12. Pathak S (2019) Improved thermal performance of annular fin-shell tube storage system using magnetic fluid. Applied Energy 239: 15241535.

13. Pathak S (2017) Magnetic Fluid Based High Precision Temperature Sensor. IEEE Sensors Journal 17(9): 2670-2675.

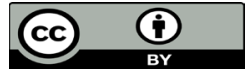

This work is licensed under Creative Commons Attribution 4.0 License

To Submit Your Article Click Here:

Submit Artic

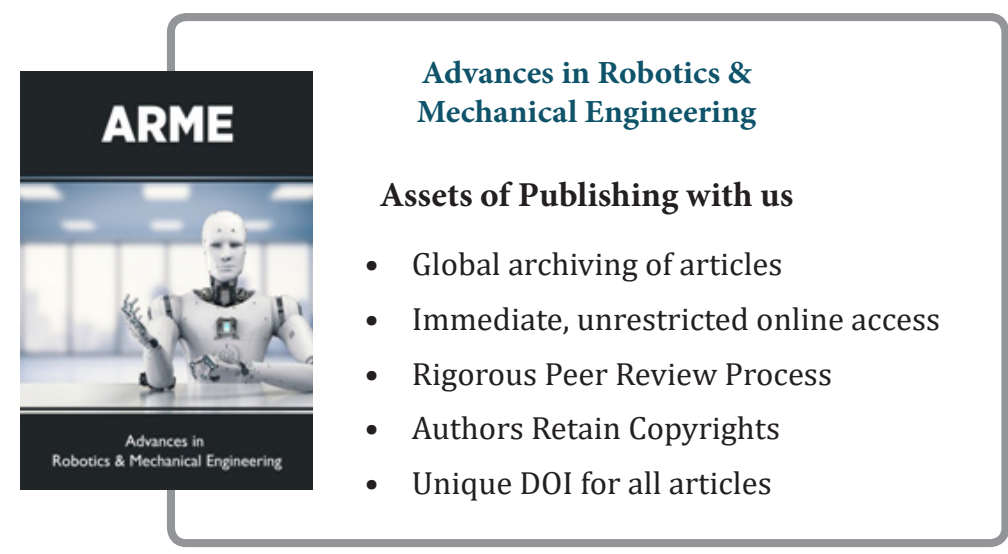

